\title{
Supporting Information \\ Quantum correlated plasmons and their tunability in undoped and doped \\ Mott-insulator cuprates
}

Xinmao Yin ${ }^{1,3}$, Chi Sin Tang ${ }^{1,2,3}$, Shengwei Zeng ${ }^{1,4}$, Teguh Citra Asmara ${ }^{1,3,4}$, Ping

Yang $^{3}$, M. Avicenna Naradipa ${ }^{1}$, Paolo E. Trevisanutto ${ }^{3,6}$, Tomonori Shirakawa ${ }^{7}$,

Beom Hyun Kim ${ }^{7}$, Seiji Yunoki ${ }^{7}$, Mark B. H. Breese ${ }^{1,3,4}$, Thirumalai Venkatesan ${ }^{1,2,4,5}$, Andrew T. S. Wee $1,2,6$, Ariando Ariando ${ }^{1,2,4, *}$, Andrivo Rusydi 1,2,3,4,*

\section{Affiliations}

${ }^{1}$ Department of Physics, Faculty of Science, National University of Singapore, 117542,

Singapore.

${ }^{2}$ NUS Graduate School for Integrative Sciences and Engineering, National University of

Singapore, 117456, Singapore

${ }^{3}$ Singapore Synchrotron Light Source, National University of Singapore, Singapore 117603

${ }^{4}$ NUSNNI-NanoCore, National University of Singapore, Singapore 117576

${ }^{5}$ Department of Electrical and Computer Engineering, National University of Singapore,

Singapore 117583

${ }^{6}$ Centre for Advanced 2D Materials and Graphene Research Centre, National University of

Singapore, Singapore 117546

${ }^{7}$ Computational Condensed Matter Physics Laboratory, RIKEN, Wako, Saitama 351-0198, Japan

*Correspondence to: A.R. (phyandri@nus.edu.sg) or A (phyarian@nus.edu.sg) 
The Supporting Information includes:

S1. High-resolution X-ray Diffraction measurements

S2. Spectroscopic Ellipsometry measurements

S3. Carrier concentration per unit cell in YLBLCO

Number of Pages: 10

Number of Figures: 2

Number of Tables: 2 


\section{Supplementary Methods:}

S1. High-resolution X-ray Diffraction measurements. To obtain the crystal structure of $\mathrm{Y}_{0.38} \mathrm{La}_{0.62}\left(\mathrm{Ba}_{0.82} \mathrm{La}_{0.18}\right)_{2} \mathrm{Cu}_{3} \mathrm{O}_{\mathrm{y}}$ (YLBLCO), high-resolution X-ray diffraction were performed from the X-ray Demonstration and Development (XDD) beamline at the Singapore Synchrotron Light Source (SSLS). Reciprocal space mappings (RSMs) are measured by coplanar diffraction geometry. The lattice constants of YLBLCO are based on that of $\mathrm{LaAlO}_{3}(\mathrm{LAO})$ substrate. The RSMs around of $(002)_{\mathrm{HL}},(002)_{\mathrm{KL}},(\overline{1} 03)_{\mathrm{HL}}$, and $(013)_{\mathrm{KL}}$ for sample $\mathrm{N} 2$ are shown in Figure S1. In Figure S1a and $b$, the peaks for YLBLCO layer is right below the LAO substrate peak, which means that there is no tilt between the YLBLCO layer and LAO substrate. In Figure S1c and d, the absolute value of $\mathrm{H}$ and $\mathrm{K}$ for YLBLCO peaks are equal and they are at the same height of L, which suggests that the lattice constants, $a$ and $b$ for $\mathrm{N} 2$ are equal. This indicates that the N2-YLBLCO with high quality is tetragonal. According to the RSMs measurements of other YLBLCO samples (not shown in the figures), it demonstrate that all the YLBLCO samples are tetragonal structure.

S2. Spectroscopic Ellipsometry measurements. Spectroscopic Ellipsometry has no charging issue due to its photon-in-photon-out methodology. Note that electron spectroscopic methods such as electron energy loss spectroscopy, while suitable for detecting plasmons in metals, are not appropriate for Mott-insulators due to severe charging problems. We can study simultaneously the complex dielectric response and 
dynamical loss-function of cuprates in their Mott-insulating state. Its detector only registers the dielectric signals of the sample at that particular photon energy value. It is different from that of other techniques such as photoluminescence measurement. Hence, the onset of higher harmonic process as the contribution to the respective higher-energy peaks is ruled out.

We used a photon energy range of $0.5 \mathrm{eV}-4 \mathrm{eV}$ to measure the ellipsometry parameters $\Psi$ (the ratio between the amplitude of $\mathrm{p}$ - and s-polarized reflected light) and $\Delta$ (the phase difference between of $\mathrm{p}$ - and s-polarized reflected light) with incident angles at $60^{\circ}, 65^{\circ}, 70^{\circ}$ for room-temperature and $70^{\circ}$ for low-temperature measurements. These incident angles indicate that the spectra include both in-plane and out-of-plane contributions. The optical conductivity has been extracted from the parameters $\Psi$ and $\Delta$ by utilizing an air/YLBLCO/LAO multilayer model, where the YLBLCO composed of an average homogeneous and uniform medium.

The raw data measured by ellipsometry is expressed in terms of $\Psi$ (change in intensity) and $\Delta$ (change in phase), which are defined as

$$
\tan \Psi \exp (i \Delta) \equiv \frac{r_{\mathrm{p}}}{r_{\mathrm{s}}},
$$

where $r_{\mathrm{p}}$ and $r_{\mathrm{s}}$ are the reflectivity of p- (parallel to the plane of incident) and s(perpendicular to the plane of incident) polarized light. From the Fresnel equations, these two quantities can be defined as

$$
r_{\mathrm{p}}^{i j}=\frac{n_{j} \cos \theta_{i}-n_{i} \cos \theta_{j}}{n_{j} \cos \theta_{i}+n_{i} \cos \theta_{j}}
$$

and 


$$
r_{\mathrm{s}}^{i j}=\frac{n_{i} \cos \theta_{i}-n_{j} \cos \theta_{j}}{n_{i} \cos \theta_{i}+n_{j} \cos \theta_{j}} .
$$

Here, $n$ and $\theta$ represent the refraction index and angle of incident from the surface normal, respectively. The $i$ and $j$ represent the two materials involved in the photon propagation. From here, the complex dielectric function $\varepsilon(\omega)=\varepsilon_{1}(\omega)+i \varepsilon_{2}(\omega)$ can be obtained using

$$
\sqrt{\varepsilon(\omega)}=n(\omega)
$$

where $\omega$ is the photon frequency.

To extract the $\varepsilon(\omega)$ of the YLBLCO films, the samples are modelled as having two layers: YLBLCO film on LAO substrate. According to the analysis of wave propagation through stratified media, the reflectivity (and thus $\Psi$ and $\Delta$ via Supplementary Eq. S1) of YLBLCO film on LAO substrate can be expressed as,

$$
r_{\text {multi }}=\frac{r_{\text {amb,YLBLCO }}+r_{\mathrm{YLBLCO}, \mathrm{LAO}} e^{i 2 \delta_{\mathrm{YLBLCO}}}}{1+r_{\mathrm{amb}, \mathrm{YLBLCO}} r_{\mathrm{YLBLCO}, \mathrm{LAO}} e^{i 2 \delta_{\mathrm{YLBLCO}}}},
$$

where

$$
\delta_{\mathrm{YLBLCO}}=\frac{2 \pi d_{\mathrm{YLBLCO}}}{\lambda} \sqrt{n_{\mathrm{YLBLCO}}^{2}-n_{\mathrm{amb}}^{2} \sin ^{2} \theta} .
$$

Here, the subscripts multi and amb represent the YLBLCO on LAO multilayer system and the ambient, respectively, while $\delta_{\text {YLBLCO }}$ is the change in light phase as it travels through the YLBLCO film, $d_{\text {YLBLCO }}$ is the thickness of the YLBLCO film, and $\lambda$ is the light wavelength.

The energy-dependent loss function can be extracted:

$$
\operatorname{Im}\left(-\frac{1}{\varepsilon(\omega)}\right)=\frac{\varepsilon_{2}(\omega)}{\varepsilon_{1}^{2}(\omega)+\varepsilon_{2}^{2}(\omega)},
$$

Figure S2 displays the raw data of ellipsometric parameters $\Psi$ (the ratio between the amplitude of $\mathrm{p}$ - and s-polarized reflected light) and $\Delta$ (the phase difference between of p- and s-polarized reflected light) for the zero-doped I0-YLBLCO, other hole and 
electron-doped YLBLCO, and YBCO samples. The experimental data for $\Psi$ and $\Delta$ appear significantly different at their respective angles because they are functions of both dielectric functions and incident angles. Eventually, data fitting of the ellipsometric parameters at different angles yield almost identical optical and loss-function profiles.

S3. Carrier concentration per unit cell in YLBLCO. The carrier concentration per unit cell of each YLBLCO sample can be calculated with respect to their respective volume.

$\mathrm{P} 1=3.9 \times 10^{-4}$ per unit cell

$\mathrm{P} 2=3.6 \times 10^{-3}$ per unit cell

I0-YLBLCO: 0 per unit cell (insulating sample)

$\mathrm{N} 1=6.4 \times 10^{-4}$ per unit cell

$\mathrm{N} 2=3.4 \times 10^{-3}$ per unit cell

However, we note that these rates are too low and that the doping rate of samples and the carrier concentration per unit cell may not be equivalent. In this case, the exact doping rate of each sample is hard to be quantified. Nevertheless, this does not affect our main conclusion. Carrier concentration is used to present the evolution of the doping, and that the sample with higher doping rate should have higher carrier concentration.

\section{Supplementary Figures}


(a)

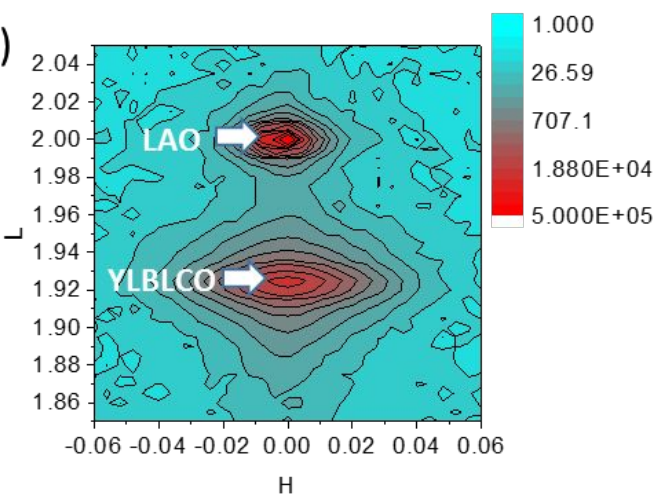

(c)

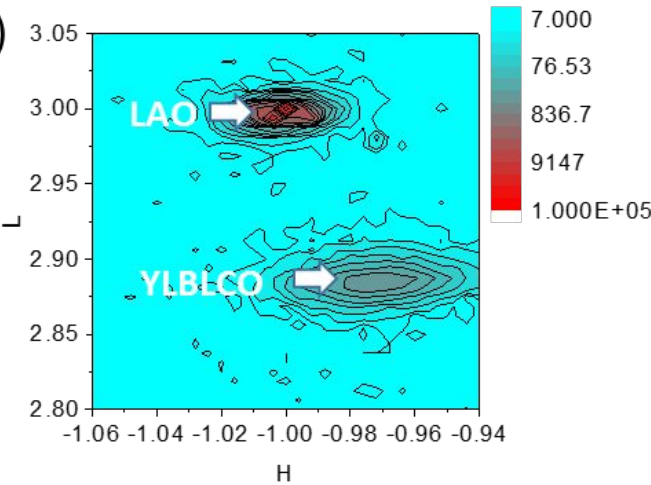

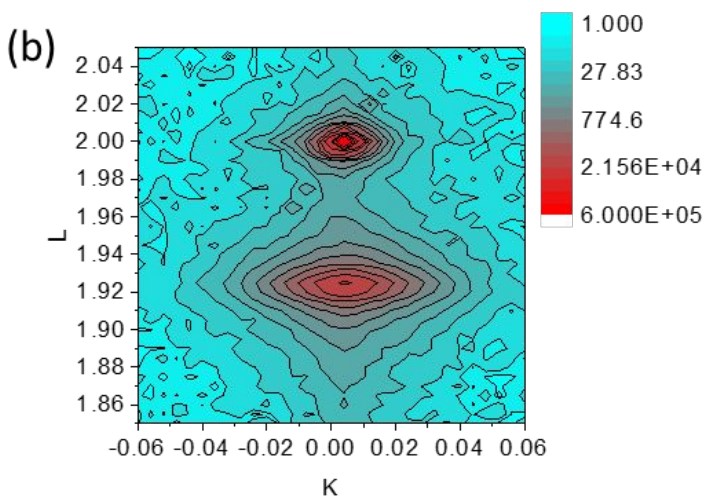

(d)

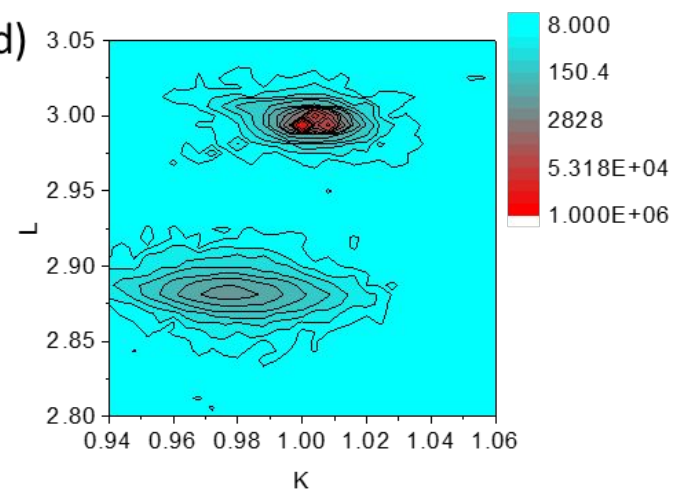

Figure S1. Reciprocal space mappings RSMs around (a) $(002)_{\mathrm{HL}},(\mathbf{b})(002)_{\mathrm{KL}}$, (c) $(\overline{1}$ 03) $)_{\mathrm{HL}}$, and (d) $(013)_{\mathrm{KL}}$ are indexed in the lattice with a, b, and c for sample N2. 

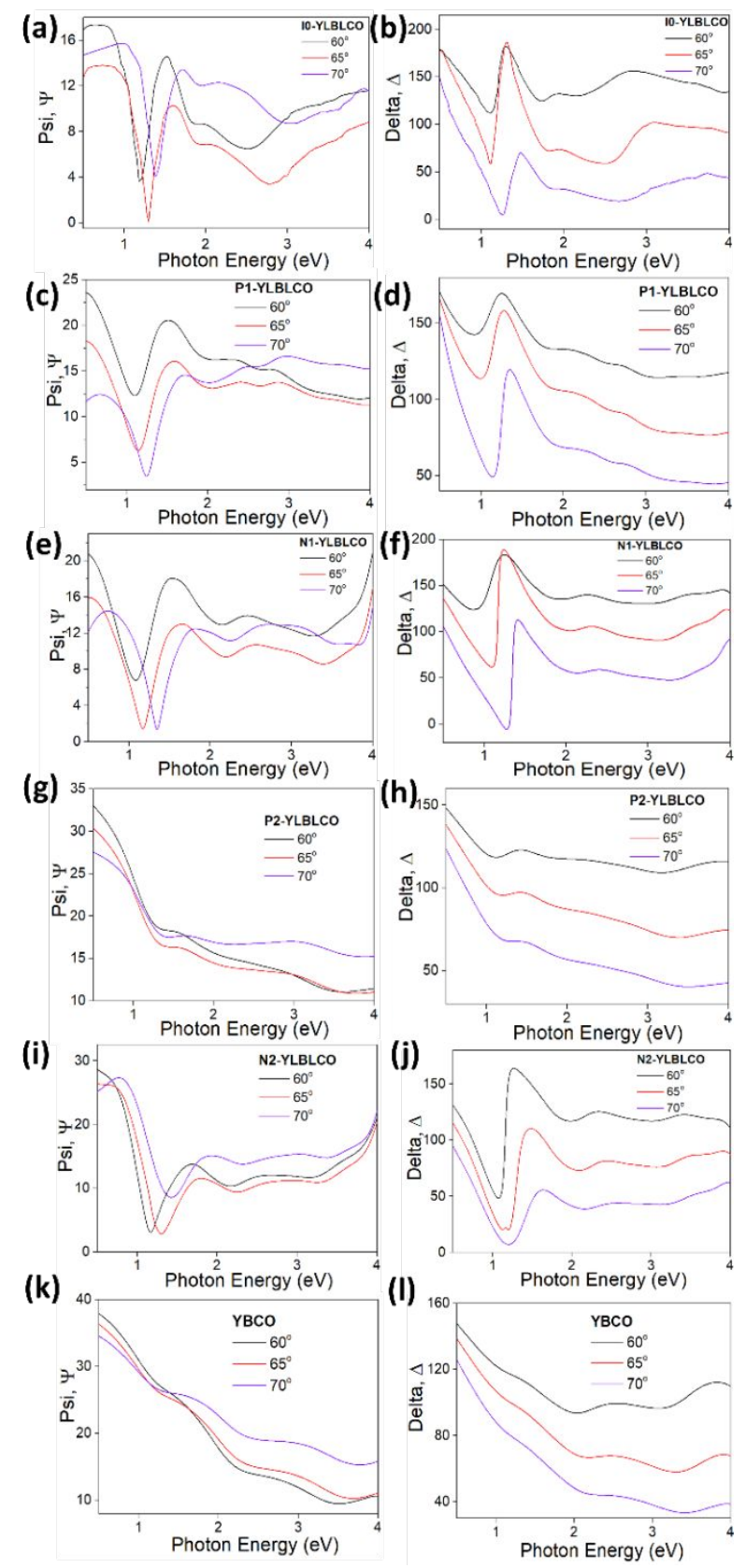

Figure S2. Raw data of the ellipsometric parameters $\Psi$ (ratio between the amplitude of p- and s-polarized reflected light) and $\Delta$ (phase difference between of p- and s-polarized reflected light) at angles $60^{\circ}, 65^{\circ}$ and $70^{\circ}$ for (a,b) I0-YLBLCO, (c,d) P1-YLBLCO, (e,f) N1-YLBLCO, (g,h) P2-YLBLCO, (i,j) N2-YLBLCO, and $(\mathbf{k}, \mathbf{l})$ YBCO. Eventually, data fitting of these ellipsometric parameters at different angles yield almost identical optical and loss-function profiles. But advanced Muller-Matrix spectroscopic ellipsometry measurements should be performed for 
future studies to reveal the intrinsic anisotropy of cuprates (details in main text).

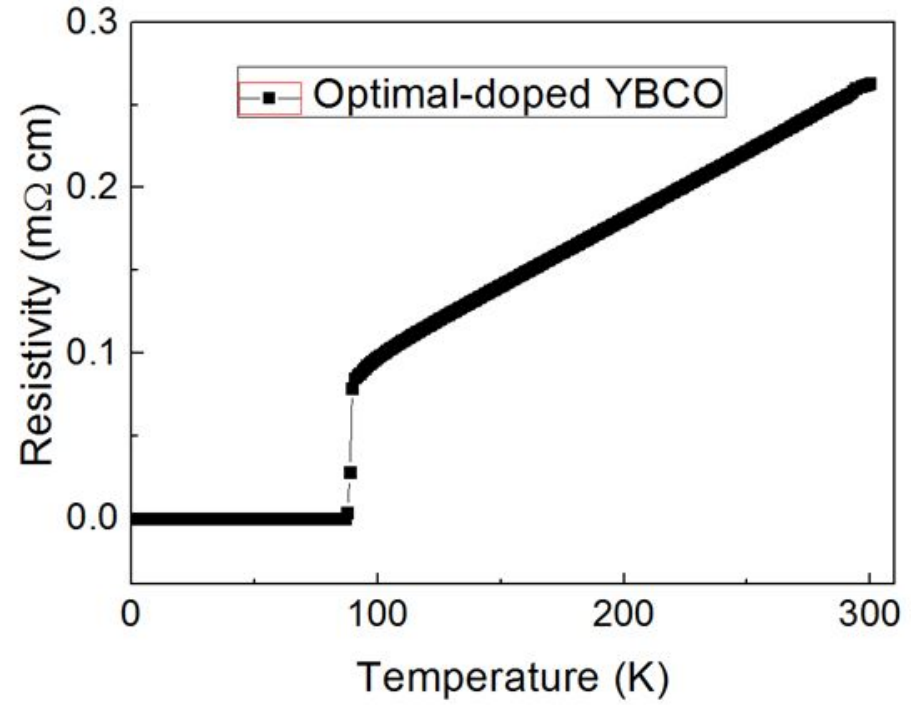

Figure S3. Temperature-dependent resistivity data of optimal-doped YBCO.

\section{Supplementary Tables}

\begin{tabular}{|c|c|c|c|c|c|c|}
\hline & \multicolumn{3}{|c|}{ (p-Type YLBLCO) } & \multicolumn{3}{|c|}{ (n-Type YLBLCO) } \\
\hline Peak & & $\begin{array}{c}\text { Energy } \\
\text { Position (eV) }\end{array}$ & $\begin{array}{c}\text { Spectral } \\
\text { Weight } \\
\text { (Voigt Profile) }\end{array}$ & & $\begin{array}{c}\text { Energy } \\
\text { Position (eV) }\end{array}$ & $\begin{array}{c}\text { Spectral Weight } \\
\text { (Voigt Profile) }\end{array}$ \\
\hline$A^{\prime}$ & \multirow{4}{*}{ I0 } & 1.01 & 0.0296 & \multirow{4}{*}{ I0 } & 1.01 & 0.0296 \\
\hline $\mathrm{B}^{\prime}$ & & 1.64 & 0.0487 & & 1.64 & 0.0487 \\
\hline$C^{\prime}$ & & 2.03 & 0.0234 & & 2.03 & 0.0234 \\
\hline$D^{\prime}$ & & 3.26 & 0.0110 & & 3.26 & 0.0110 \\
\hline$A^{\prime}$ & \multirow{4}{*}{ P1 } & 1.05 & 0.0216 & \multirow{4}{*}{$\mathbf{N} 1$} & 0.80 & 0.0522 \\
\hline $\mathrm{B}^{\prime}$ & & 1.73 & 0.0609 & & 1.78 & 0.0349 \\
\hline $\mathrm{C}^{\prime}$ & & 2.05 & 0.0188 & & 1.60 & 0.00765 \\
\hline $\mathrm{D}^{\prime}$ & & 3.55 & 0.0679 & & 3.43 & 0.0102 \\
\hline$A^{\prime}$ & \multirow{4}{*}{$\mathbf{P 2}$} & 1.01 & 0.0917 & \multirow{4}{*}{ N2 } & 0.81 & 0.0718 \\
\hline $\mathrm{B}^{\prime}$ & & 1.58 & 0.0963 & & 1.81 & 0.0846 \\
\hline$C^{\prime}$ & & 2.01 & 0.0131 & & 1.45 & 0.00477 \\
\hline $\mathrm{D}^{\prime}$ & & 3.16 & 0.0804 & & 3.56 & 0.0298 \\
\hline
\end{tabular}

Table S1. Plasmonic peak profiles of hole and electron-doped YLBLCO. 


\begin{tabular}{|c|c|c|c|c|c|c|c|c|c|}
\hline \multicolumn{5}{|c|}{ (p-Type YLBLCO) } & \multicolumn{5}{|c|}{ (n-Type YLBLCO) } \\
\hline \multirow[t]{2}{*}{ Peak } & \multicolumn{2}{|c|}{$\mathbf{A}^{\prime}$} & \multicolumn{2}{|c|}{ B' } & \multirow[t]{2}{*}{ Peak } & \multicolumn{2}{|c|}{$\mathbf{A}^{\prime}$} & \multicolumn{2}{|c|}{ B' } \\
\hline & $\begin{array}{c}\text { FWHM } \\
(\mathrm{eV})\end{array}$ & $\begin{array}{c}\text { D-Time } \\
\text { (fs) }\end{array}$ & $\begin{array}{c}\text { FWHM } \\
(\mathrm{eV})\end{array}$ & $\begin{array}{c}\text { D-Time } \\
\text { (fs) }\end{array}$ & & $\begin{array}{c}\text { FWHM } \\
(\mathrm{eV})\end{array}$ & $\begin{array}{c}\text { D-Time } \\
\text { (fs) }\end{array}$ & $\begin{array}{c}\text { FWHM } \\
(\mathrm{eV})\end{array}$ & $\begin{array}{c}\text { D-Time } \\
\text { (fs) }\end{array}$ \\
\hline I0 & 0.23 & 5.72 & 0.38 & 3.46 & I0 & 0.23 & 5.72 & 0.38 & 3.46 \\
\hline $\mathbf{P 1}$ & 0.35 & 3.76 & 11.8 & 2.48 & N1 & 0.44 & 2.99 & 0.40 & 3.29 \\
\hline $\mathbf{P 2}$ & 0.58 & 2.27 & 0.77 & 1.71 & $\mathbf{N 2}$ & 0.19 & 6.93 & 0.61 & 2.16 \\
\hline
\end{tabular}

Table S2. Change in dephasing profiles at different levels of electron/hole doping. 